\title{
Label-free human chorionic gonadotropin detection at picogram levels using oriented antibodies bound to graphene screen-printed electrodes
}

\author{
Sofia Teixeira, Robert S. Conlan, O. J. Guy and M. Goreti F. Sales
}

Human chorionic gonadotropin (hCG) is a key diagnostic marker of pregnancy and an important biomarker for cancers in the prostate, ovaries and bladder and therefore of great importance in diagnosis. For this purpose, a new immunosensor of screen-printed electrodes (SPEs) is presented here. The device was fabricated by introducing a polyaniline (PANI) conductive layer, via in situ electropolymerization of aniline, onto a screen-printed graphene support. The PANI-coated graphene acts as the working electrode of a three terminal electrochemical sensor. The working electrode is functionalised with anti-hCG, by means of a simple process that enabled oriented antibody binding to the PANI layer. The antibody was attached to PANI following activation of the $-\mathrm{COOH}$ group at the Fc terminal. Functionalisation of the electrode was analysed and optimized using Electrochemical Impedance Spectroscopy (EIS). Chemical modification of the surface was characterised using Fourier transform infrared, and Raman spectroscopy with confocal microscopy. The graphene-SPE-PANI devices displayed linear responses to hCG in EIS assays from 0.001 to $50 \mathrm{ng} \mathrm{mL}^{-1}$ in real urine, with a detection limit of $0.286 \mathrm{pg} \mathrm{mL}^{-1}$. High selectivity was observed with respect to the presence of the constituent components of urine (urea, creatinine, magnesium chloride, calcium chloride, sodium dihydrogen phosphate, ammonium chloride, potassium sulphate and sodium chloride) at their normal levels, with a negligible sensor response to these chemicals. Successful detection of hCG was also achieved in spiked samples of real urine from a pregnant woman. The immunosensor developed is a promising tool for point-of-care detection of hCG, due to its excellent detection capability, simplicity of fabrication, low-cost, high sensitivity and selectivity.

\section{Introduction}

Immunoassay techniques, using a specific molecular interaction between antibody and antigen, have become the dominant clinical test methods for antigen detection. Alternative immunoassay detection methods include radioimmunoassay, enzyme-linked immunosorbent assay (ELISA), fluoroimmunoassay, chemiluminescence immunoassay, and electrochemiluminescence immunoassay. ${ }^{1}$ Despite their sensitivity, precision and selectivity, these techniques suffer from several drawbacks such as radiation hazards, long test times, high costs, and the need for qualified personnel and sophisticated instrumentation to perform the assays. Furthermore, existing diagnostic tests (e.g., microtiter-plate ELISAs) may not be sensitive enough for screening a wide range of proteins at low concentrations, with optical detection suffering from interference from other luminescence molecules. Also, single-analyte immunoassays are not fast enough for high-volume throughput. ${ }^{1}$ New immunoassay systems for sensitive, selective, low-cost, multi-analyte and high-throughput immunoassay have therefore attracted considerable interest, leading to the integration of immune-reactions into electrochemical biosensors.

An electrochemical immunosensor is, by IUPAC definition, a self-contained integrated device, which produces (semi-)quantitative analytical information using an antibody as a biological recognition element that is in direct contact with an electrochemical transduction element. ${ }^{2}$ Electrochemical immunosensors combine the specificity of an immunological reaction, the possibility of application in point-of-care testing - due to their easy miniaturization and the low cost of typical electrochemical biosensors. ${ }^{3,4}$ Compared with traditional immunoassay methods, electrochemical immunosensors are specific, selective, highly sensitive, simple and convenient, can offer 
multi-target analyses, use simple instrumentation, allow rapid analysis, can be miniaturized, and can be manufactured using advanced fabrication technologies.,

In electrochemical immunosensing, direct detection of antibody-antigen recognition is usually not possible and electrochemically redox couples are often employed. ${ }^{7}$ Colloidal gold/silver, semiconductor nanoparticles and marker loaded nanocarriers (carbon nanotubes, apoferritin, silica nanoparticles, and liposome beads) have all been used as labels. ${ }^{8}$ However, this is a complex and quite expensive procedure.

In contrast, electrochemical impedance spectroscopy (EIS) can be used to directly measure the effect of antigen binding on the resistance to charge-transfer across the electrode surface.

Electrochemical immunosensors using advances in nanomaterials are increasingly being used for biosensing applications. ${ }^{9}$ In this context, carbon-based nanomaterials are gaining interest as an alternative to gold electrodes. Different carbon nanostructures, with different inherent electrical properties have been used in electrochemical immunosensors including $\mathrm{CNTs}^{\mathbf{1 0}}$ and more recently (since 2005) graphene. ${ }^{\mathbf{1 1}}$

Graphene displays a combination of interesting properties, such as a large surface area, high electrical conductivity and biocompatibility. ${ }^{\mathbf{1 2}-19}$ Heterogeneous electron transfer (the transfer of electrons between graphene and molecules in solution necessary for the oxidation/reduction of said molecules) occurs at the edges of graphene or at defects in the basal plane. ${ }^{7}$ Additional defects are also introduced by functionalization, required to immobilize biomolecules, including antibodies, on to graphene. The antibodies act as biological receptors in immunosensors. This can affect the performance of the sensor. An alternative way of attaching the bioreceptor antibodies to the sensor electrode is through grafting to an intermediate layer such as pyrene ${ }^{20}$ or polyaniline. ${ }^{21}$ This avoids the introduction of defects into the graphene substrate and preserves graphene's electrical characteristics. ${ }^{22}$ Intermediary pyrene or polyaniline layers allow efficient electron transfer to the graphene electrode. Correct orientation of the antibody on the PANI-graphene support is crucial to ensure that the two binding sites in each antibody are free for antigen binding and that antibody activity is preserved after grafting.

Thus, this work proposes a new immunosensor set-up, where the antibody has been directly attached to a PANImodified screen-printed graphene electrode, and used to detect human chorionic gonadotropin (hCG) using label-free EIS measurements. Human chorionic gonadotropin (hCG) detection, a $37 \mathrm{kDa}$ glycoprotein hormone, is a well-known and important diagnostic marker of pregnancy, but may also act as a tumour marker for certain cancers. The concentrations of hCG in serum or urine emanating from the tumours are very low. ${ }^{23,2425}$ Thus, the detection method for hCG in serum or urine should ensure that trace amounts of hCG are detectable.

At present, conventional methods for determining hCG are immunoradiometric assays (IRMAs) and enzyme-linked immunosorbent assays (ELISA). ${ }^{26}$ IRMA is extremely sensitive, but involves radiation hazards, complicated washing procedures and is expensive, while the ELISA is less sensitive but time-consuming. Various electrochemical immunosensors for
hCG measurements have also been reported recently, but only a few of these use graphene. ${ }^{27-30}$ The overall process involved in the assembly of these immunosensors and the relevant chemical course/data for hCG determination have been resumed in Table 1. Overall, graphene is employed within a quite complex chemistry to produce specific composite nanomaterials. These nanomaterials are in turn involved in the assembly of immunosensors including several consecutive layers, increasing the risk of failure of the device in routine testing. Furthermore, chemically modified positions in graphene may hinder the excellent electronic transfer ability of this carbon matrix for changing the carbon hybridization from $\mathrm{sp}^{2}$ to $\mathrm{sp}^{3}$, thereby interrupting the path of conjugated $\pi$ electrons. Finally, the overall processes for hCG detection are especially complex when labeled nanomaterials are employed, requiring as well longer times for reaching a response.

The immunosensor, reported in this paper, is a simple device, produced via modification of a disposable graphene-SPE surface with an amine layer, which preserves the chemical structure of graphene and allows subsequent binding of the bioreceptor antibody, anti-hCG. The amine layer is obtained by in situ electropolymerization of aniline on the graphene electrode and these amine groups can react with the-COOH groups in the Fc region of the antibody, activated by carbodiimide chemistry, to ensure successful antibody binding. The antibody is bound to the PANI through its Fc terminal (thus allowing the paratope regions free to bind to the antigen epitopes). The resulting biosensor has been evaluated using EIS, and utilised in the detection of hCG in biological samples.

\section{Experimental section}

\subsection{Reagents and solutions}

All chemicals used were of analytical grade and water was deionized. Potassium hexacyanoferrate(III) $\left(\mathrm{K}_{3}\left[\mathrm{Fe}(\mathrm{CN})_{6}\right]\right)$ and potassium hexacyanoferrate(II) $\left(\mathrm{K}_{4}\left[\mathrm{Fe}(\mathrm{CN})_{6}\right]\right)$ trihydrate were obtained from Riedel Haen; $N$-hydroxysuccinimide (NHS) was obtained from Fluka; $N$-(3-dimethylaminopropyl)- $N^{\prime}$-ethylcarbodiimide hydrochloride (EDAC), bovine serum albumin (BSA), and aniline were obtained from Sigma; phosphate buffered saline (PBS) tablets were obtained from Amresco. The hCG protein was purchased from Abcam (UK). The anti-hCG antibody was supplied by Ig Innovations.

A $0.1 \mathrm{M}$ aniline solution was prepared in $2.5 \mathrm{M} \mathrm{H}_{2} \mathrm{SO}_{4}$. PBS solution was prepared by dissolution of 1 tablet (1814.5$2005.5 \mathrm{mg}$ per tab) of PBS in $200 \mathrm{~mL}$ of D.I. water. EDAC, NHS and antibody solutions were prepared in this buffer. Synthetic urine was prepared by forming a solution of urea, creatinine, magnesium chloride, calcium chloride, sodium dihydrogen phosphate, ammonium chloride, potassium sulphate and sodium chloride.

\subsection{Apparatus}

Electrochemical measurements were conducted with a potentiostat/galvanostat from Metrohm Autolab PGSTAT302N, with a Frequency Response Analysis (FRA) module, controlled by Nova 


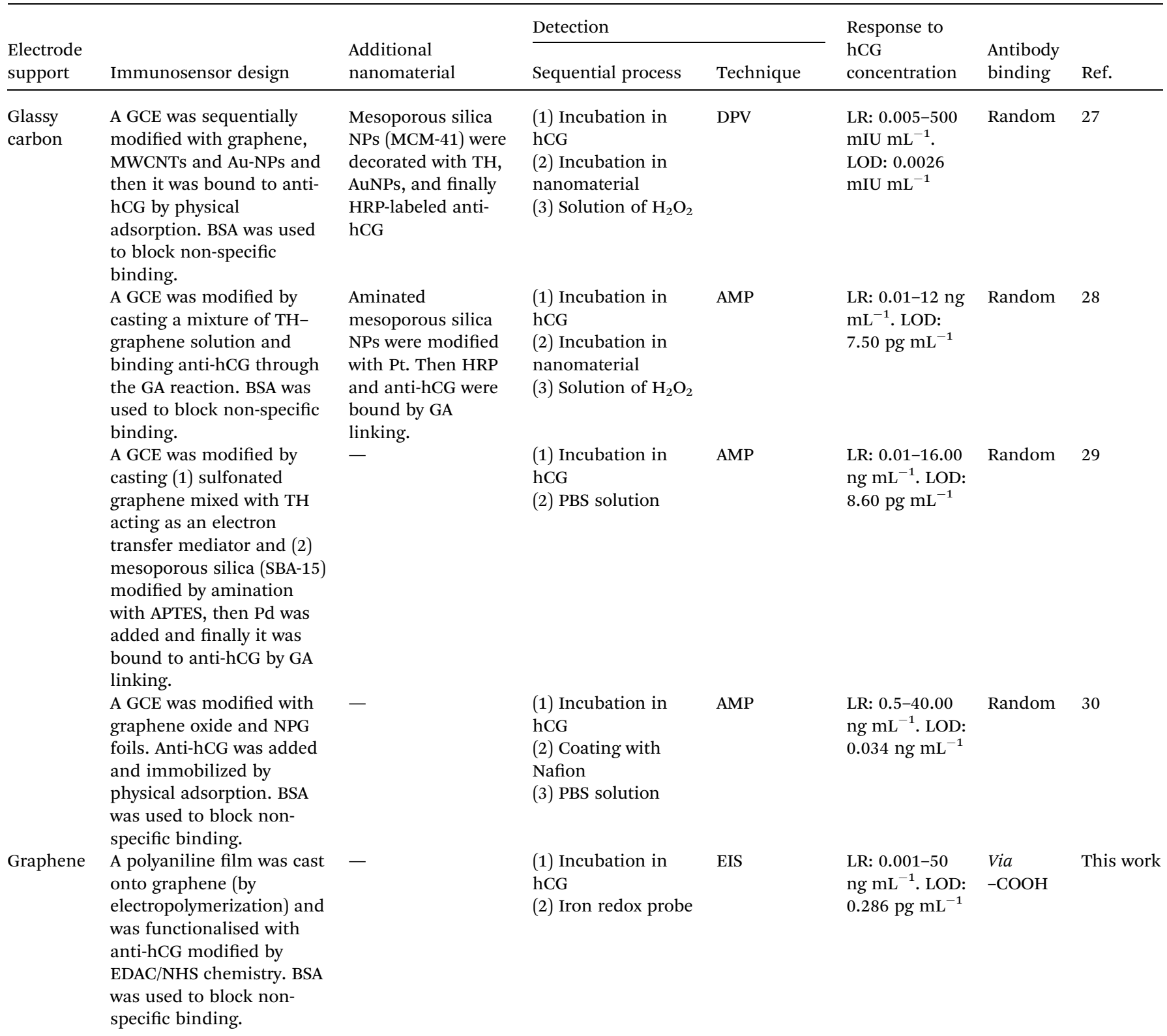

\begin{abstract}
${ }^{a}$ AMP: amperometry; AP: alkaline phosphatase; APTES: 3-aminopropyltrimethoxy-silane; DPV: differential pulse voltammetry; EDAC: $N$-(3dimethylaminopropyl)- $N^{\prime}$-ethylcarbodiimide; EIS: electrochemical impedance spectroscopy; GA: glutaraldehyde; GCE: glassy carbon electrode; GPMS: glycidoxypropyltrimethoxysilane; HRP: horseradish peroxidase; MWCNTs: multiwall carbon nanotubes; NHS: $N$-hydroxysuccinimide; NPG: nanoporous gold; NPs: nanoparticles; SPDP: succinimidyl-6-[3'-(2-pyridyldithio)-propionamido]hexanoate; TH: thionine.
\end{abstract}

software. Graphene-SPEs were purchased from DropSens (DRP$110 \mathrm{GPH}$ ), having a counter electrode of platinum (Pt), a reference electrode of $\mathrm{Ag} / \mathrm{AgCl}$, and a working electrode of printed graphene of $4 \mathrm{~mm}$ diameter. The graphene-SPEs were interfaced with the potentiostat/galvanostat via a switch box, also from DropSens (DRP-DSC). Raman measurements were performed using a Thermo Scientific DXR Raman microscope system with a $100 \mathrm{~mW} 532 \mathrm{~nm}$ excitation laser, and spectra were recorded using $5 \mathrm{~mW}$ power and a $50 \mu \mathrm{m}$ pinhole aperture. Fourier transform infrared spectroscopy (FTIR) measurements were performed using a Thermo Scientific Smart iTR Nicolet
iS10, coupled to the Attenuated Total Reflectance (ATR) smart accessory, also from Thermo Scientific.

\subsection{Procedures for immunosensor assembly}

The polyaniline (PANI) film was obtained by electropolymerization of aniline on the graphene-SPE surface using cyclic voltammetry (CV). The three-electrodes of the SPE were covered by a solution of $0.1 \mathrm{M}$ aniline and a potential sweep applied from $-0.1 \mathrm{~V}$ to $1.2 \mathrm{~V}$, at a sweep rate of $50 \mathrm{mV} \mathrm{s}^{-1}$, for 20 cycles. 
In parallel, an antibody solution of $200 \mu \mathrm{g} \mathrm{mL} \mathrm{m}^{-1}$ was mixed with $25 \mathrm{mmol} \mathrm{L}^{-1}$ EDAC and $50 \mathrm{mmol} \mathrm{L}^{-1}$ of NHS, for 2 hours. A drop of this resulting solution was then placed on the PANI/ graphene-SPE surface. After 2 hours, at room temperature, the electrode was rinsed away and replaced for BSA solution $(0.5 \mathrm{mg}$ $\mathrm{mL}^{-1}$ in PBS buffer), for 30 minutes. The immunosensor was then washed with PBS buffer.

\subsection{Human chorionic gonadotropin binding}

hCG binding to the antibody on the immunosensor was achieved by placing a drop of the hCG solution on the sensor surface. Different concentrations of hCG solution, ranging from 0.01 to $5 \mathrm{ng} \mathrm{mL} \mathrm{me}^{-1}$, were prepared by dilution of the $25 \mathrm{ng} \mathrm{m \textrm {m } ^ { - 1 }}$ standard hCG solution in PBS or synthetic urine. hCG was also detected in real urine samples from a pregnant woman. A period of 15 minutes was allowed for antigen/antibody binding. This was followed by PBS washing prior to redox probe EIS measurements.

\subsection{Electrochemical assays}

$\mathrm{CV}$ measurements were conducted in $5.0 \mathrm{mmol} \mathrm{L}^{-1}$ of $\left[\mathrm{Fe}(\mathrm{CN})_{6}\right]^{3-}$ and $5.0 \mathrm{mmol} \mathrm{L}^{-1}$ of $\left[\mathrm{Fe}(\mathrm{CN})_{6}\right]^{4-}$, prepared in PBS buffer, $\mathrm{pH}$ 7.4. In $\mathrm{CV}$ assays the potential was scanned from $-0.7 \mathrm{~V}$ to $+0.7 \mathrm{~V}$, at $50 \mathrm{mV} \mathrm{s}^{-1}$. All assays were conducted in triplicate.

EIS assays were also conducted in triplicate with the same redox couple $\left[\mathrm{Fe}(\mathrm{CN})_{6}\right]^{3-/ 4-}$ at a standard potential of $+0.10 \mathrm{~V}$, using a sinusoidal potential perturbation with an amplitude of $100 \mathrm{mV}$ and a frequency equal to $100 \mathrm{~Hz}$, logarithmically distributed over a frequency range of $10 \mathrm{~Hz}-0.001 \mathrm{~Hz}$. The impedance data were fitted to a Randles equivalent circuit using the Nova Software.

The immunosensor response to varying hCG concentrations was assessed by EIS measurements. The concentrations of hCG

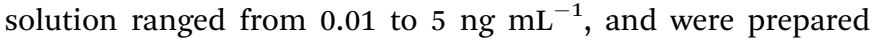
either in PBS buffer pH 7 or in synthetic urine. The limit of detection (LOD) was calculated as $3 \sigma / S$, where $S$ is the slope of the linear calibration plot and $\sigma$ the standard deviation of EIS blank signals (obtained in the absence of hCG).

\section{Results and discussion}

\subsection{Immunosensor assembly}

A schematic of the functionalisation process for the immunosensor assembly can be seen in Fig. 1 . The first stage in the sensor fabrication was modification of the screen-printed graphene electrode with a thin film of polyaniline, thus yielding surface amine groups on the PANI-graphene electrode (Fig. 1(ii)). These amine groups are used to bind the anti-hCG to the modified graphene SPE. A PANI film was formed on the graphene SPE via coating the electrode in a solution of aniline and subsequent electropolymerization of aniline to form a conductive polymer layer over the nanostructured graphene support. ${ }^{31-33}$ This PANI layer is an electrically conductive layer and is able to transport electrical carriers to the graphene SPE. ${ }^{\mathbf{3 4}}$ Of the several conductive polymers that have been employed in

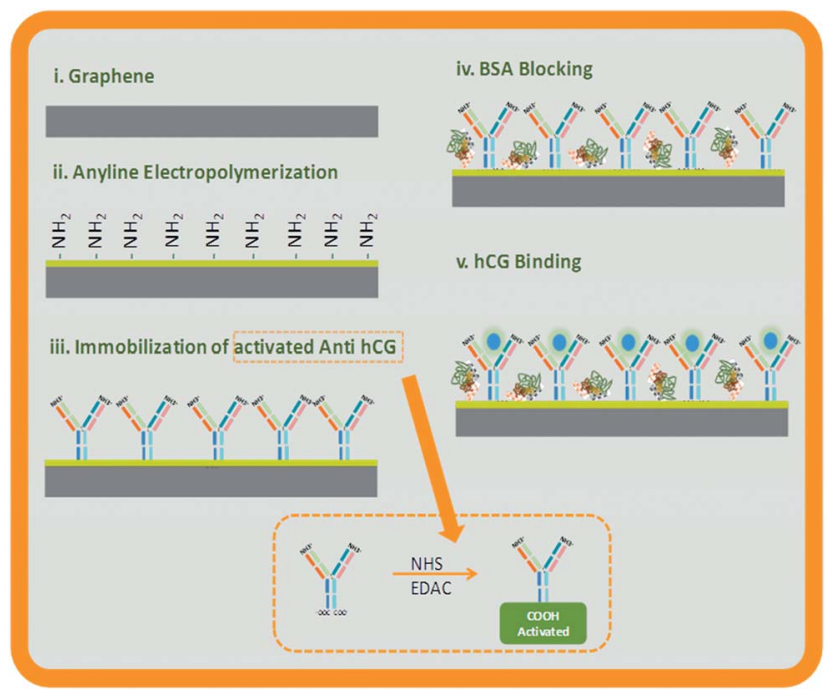

Fig. 1 Schematic illustration of the immunosensor assembly.

electrochemical applications, PANI was selected because of its simple synthesis and good stability. ${ }^{35}$ The requirement for graphene oxidation prior to electropolymerization of aniline was investigated $^{36}$ and no significant differences were observed between standard and oxidised PANI/graphene-SPE.

The next stage of the sensor fabrication was antibody binding to the PANI layer. Antibodies have several functional groups that facilitate adsorption to PANI, meaning that the antibody solution could simply be deposited on this layer. However, this procedure would lead to random positioning of the antibodies on the surface. Consequently, many antibodies could be deactivated or partially deactivated with respect to antigen binding, through stereochemical constraints.

Thus, covalent binding of the antibody to the PANI-graphene SPE was performed in order to achieve a more stable and homogenous orientation of the antibodies. This covalent binding can be accomplished through the $-\mathrm{COOH}$ terminated Fc site of the antibody. Attachment via the Fc site ensures that both $\mathrm{F}_{\mathrm{ab}}$ sites are free for subsequent antigen binding. Antibodies were first activated by EDAC/NHS chemistry, forming a highly reactive $O$-acylisourea intermediate that reacted rapidly with NHS to produce a stable succinimidyl ester. ${ }^{37}$ This ester then undergoes a nucleophilic substitution reaction with the amine groups on the PANI, leading to the formation of an antibody grafted PANI layer (Fig. 1(iii)).

The final stage of the assembly was devoted to blocking any free amine sites which could otherwise allow non-specific binding to biomolecules other than hCG. Amino groups were blocked by adding BSA, a simple and low-cost protein, to the sensor surface. BSA was adsorbed to regions of non-specific protein binding, preventing subsequent binding of the compounds different than HCG. At the same time, this reaction deactivated the carboxylic functions that remained active after antibody binding, also contributing to eliminating sideresponses from the immunosensor. 


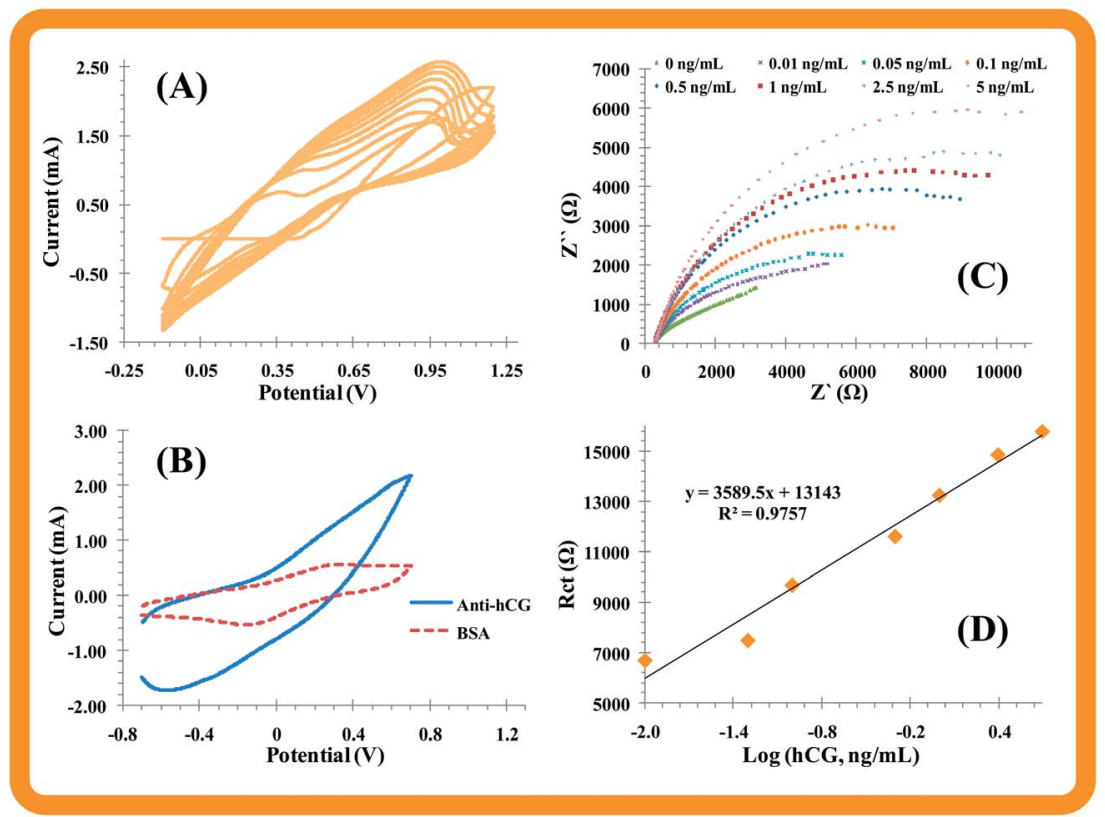

Fig. 2 CV data taken at the assembly of the immunosensor (A and B) and EIS spectra of calibration (C) along with its calibration plot (D). (A) Synthesis of polyaniline on the graphene-SPE electrode (10 cycles were performed with a scan rate of $50 \mathrm{mV} \mathrm{s}{ }^{-1}$ ); (B) CV records after modification of PANI/graphene-SPE with antibody and BSA; (C) Nyquist plots of the BSA/anti-hCG/PANI/graphene-SPE sensor, previously incubated in increasing concentrations of $\mathrm{hCG}$, obtained in $5.0 \mathrm{mM}\left[\mathrm{Fe}(\mathrm{CN})_{6}\right]^{3-} / 5.0 \mathrm{mM}\left[\mathrm{Fe}(\mathrm{CN})_{6}\right]^{4-} / \mathrm{PBS}$ buffer $\mathrm{pH} 7.4$; (D) the $R_{\mathrm{ct}}$ values of the previous calibration plotted against log hCG concentration.

\subsection{Electrochemical follow-up of the immunosensor}

The chemical modifications taking place at the graphene surface were monitored using CV and EIS (Fig. 2), characterising changes in electron transfer properties against the redox probe. ${ }^{38}$ The EIS data were analyzed by Nyquist plots. Nyquist plots showed the frequency response of the electrode/electrolyte system and area plot of the imaginary component $\left(\mathrm{Z}^{\prime \prime}\right)$ of the impedance against the real component $\left(Z^{\prime}\right)$. The charge-transfer resistance $\left(R_{\mathrm{ct}}\right)$ at the electrode surface is given by the semicircle diameter obtained in EIS and can be used to define the interface properties of the electrode. The graphene surface showed a small semicircle at the Nyquist plot, which indicated the presence of a very fast electron-transfer process. This process had no diffusion limited step, which suggests a non-Faradaic process.

$\mathrm{CV}$ analysis of the formation of a PANI layer on graphene, via electropolymerization, yielded a decrease in current with an increasing number of cycles (Fig. 2A). After 10 cycles, the current density of the oxidation peak was greatly reduced, thus confirming the formation of a PANI film. Electrochemical data, obtained by using an iron redox probe with the newly formed

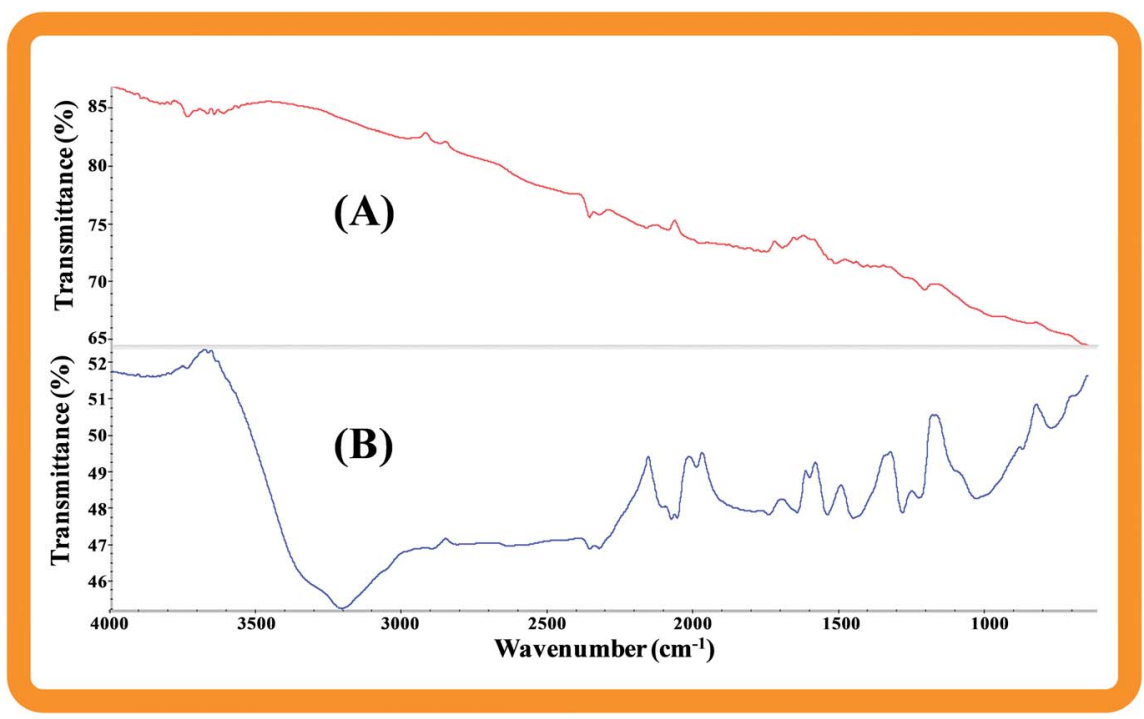

Fig. 3 FTIR spectra of modified graphene-SPE before and after polyaniline. 


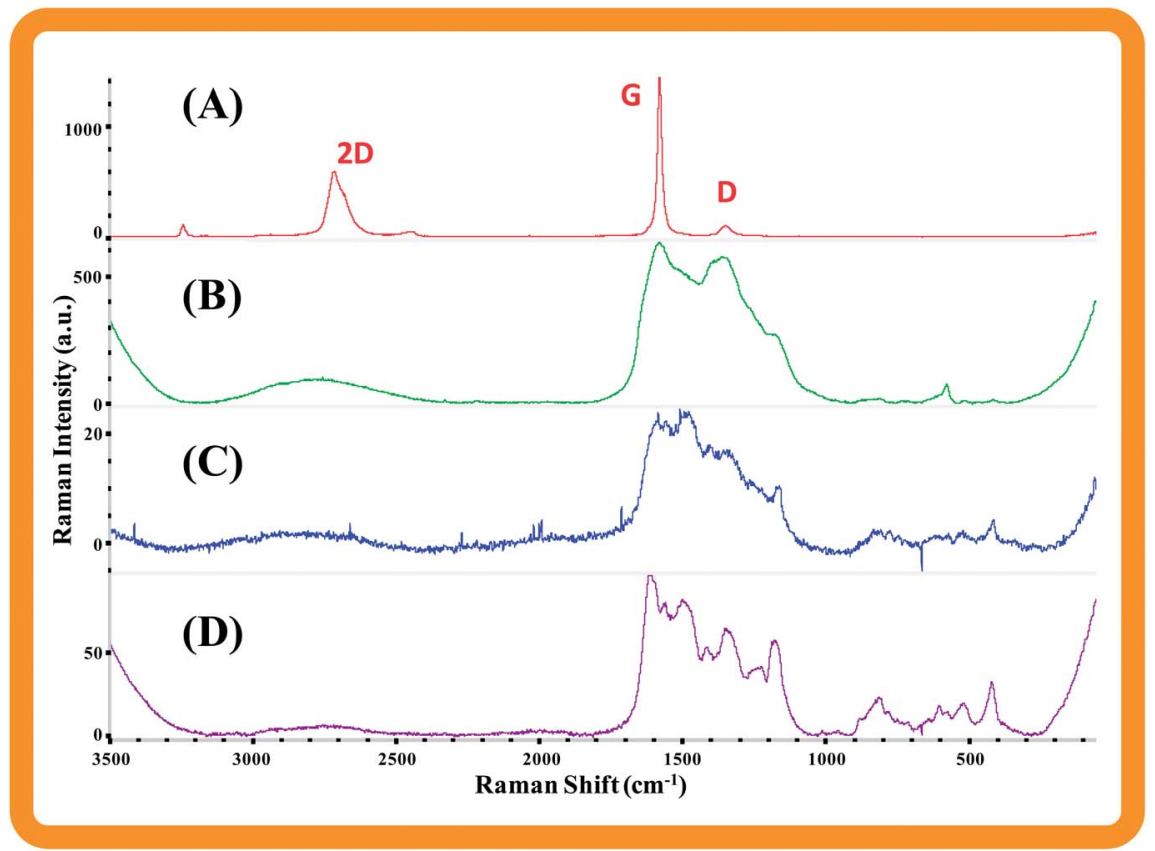

Fig. 4 Raman spectra of (A) graphene-SPE, (B) PANI/graphene-SPE, (C) anti-hCG/PANI/graphene and (D) BSA/anti-hCG/PANI/graphene-SPE.

PANI layer, showed Nyquist plots where the $R_{\mathrm{ct}}$ increased compared to unmodified graphene which was in agreement with the CV data obtained at the electropolymerization stage.

Subsequent antibody and BSA binding (both proteins) promoted a substantial increase in the barrier to the redox probe accessing the PANI/graphene-SPE surface, thus leading to an additional increase in $R_{\mathrm{ct}}$. CV assays supported the results of EIS studies (Fig. 2B), with decreased current peaks after protein binding and an increase in the peak-to-peak potential separation.

\subsection{Qualitative analysis of the immunosensor surface}

Fig. 3 shows the FTIR spectra of the graphene-SPE before any modification (A) and after coating the electrode through electropolymerisation to form the PANI/graphene-SPE (B). The graphene-SPE spectrum acted here as a control reference spectrum, from which changes related to the PANI layer in the FTIR spectrum of the PANI-graphene-SPE can clearly be distinguished.

Overall, the introduction of the PANI film on graphene gave rise to a wide range of absorption bands/peaks not present in the control sample spectrum, thus confirming the presence of the PANI layer. The two bands in the vicinity of $1500 \mathrm{~cm}^{-1}$ and $1600 \mathrm{~cm}^{-1}$ were assigned to the 6 carbon stretching modes of the bezenoid (B) and quinoid (Q) rings, respectively. ${ }^{39,40}$ The peaks in the vicinity of 1250 and $750 \mathrm{~cm}^{-1}$ were assigned to vibrations associated with the $\mathrm{C}-\mathrm{N}$ stretching vibration of aromatic amine and $\mathrm{C}-\mathrm{H}$ vibrations. ${ }^{\mathbf{4 1}}$ The absorption peak near $1150 \mathrm{~cm}^{-1}$ could be assigned to $\mathrm{B}-\mathrm{NH}-\mathrm{B}$ and $\delta(\mathrm{C}-\mathrm{H})$ vibrations. $^{42}$

Raman spectroscopic measurements (Fig. 4) were also performed after each stage of the immunosensor functionalization process. The spectra of the control graphene-SPE showed three Raman bands at 1350, 1600 and $2700 \mathrm{~cm}^{-1}$ that were assigned to the well-documented D, G and $2 \mathrm{D}$ bands in standard graphene. The $\mathrm{G}$ peaks represent the in-plane bond-stretching vibrations of $\mathrm{sp}^{2}$-hybridized carbon atoms, while the $\mathrm{D}$ peak is related to the vibrations of the carbon atoms of dangling bonds or $\mathrm{sp}^{3}$-hybridised carbon atoms of disordered and/or defected graphite. ${ }^{43}$ The intensity ratio of $I_{\mathrm{G}} / I_{\mathrm{D}}$ bands is typically used for quantifying the defect density in graphene, and the small ratio extracted from Fig. 4 indicates significant disorder, arising from structural defects, despite recent evidence that the $I_{\mathrm{G}}$ intensity increases with increasing laser excitation energy. ${ }^{\mathbf{4 4 4 5}}$ Since the intensity of the $\mathrm{G}$ band increases almost linearly as the graphene thickness increases, the ratio of $I_{\mathrm{G}} / I_{2 \mathrm{D}}$ could be used to determine the number of layers in graphene. ${ }^{46,47}$ The ratio of $I_{\mathrm{G}} /$ $I_{2 \mathrm{D}}$ obtained was $\sim 0.4$, this showing that the graphene is just a few layers thick (less than 5).

After electrochemical polymerization of aniline, all of the Raman spectra showed distinct bands attributable to PANI [45], which overlap the characteristic bands of standard graphene (at 1300 and $1600 \mathrm{~cm}^{-1}$ ). The $\mathrm{sp}^{3}$ peak of graphene becomes more intense and broad after PANI deposition. ${ }^{36}$ This broadening of the $\mathrm{sp}^{3}$ peak gives a significant increase in the intensity ratio of D/G bands.

Compared with graphene, the $\mathrm{sp}^{3}$ peak of PANI showed a slight shift to higher frequencies, probably due to the electrostatic interaction between aniline monomers and grapheneSPE, both displaying aromatic features. Overall, the obtained Raman spectra indicated the successful modification of the graphene-SPE, which considering the chemical modifications implied should suggest the formation of a PANI film.

Subsequent antibody and BSA binding introduced an additional increase in the Raman intensity, related to fluorescence 


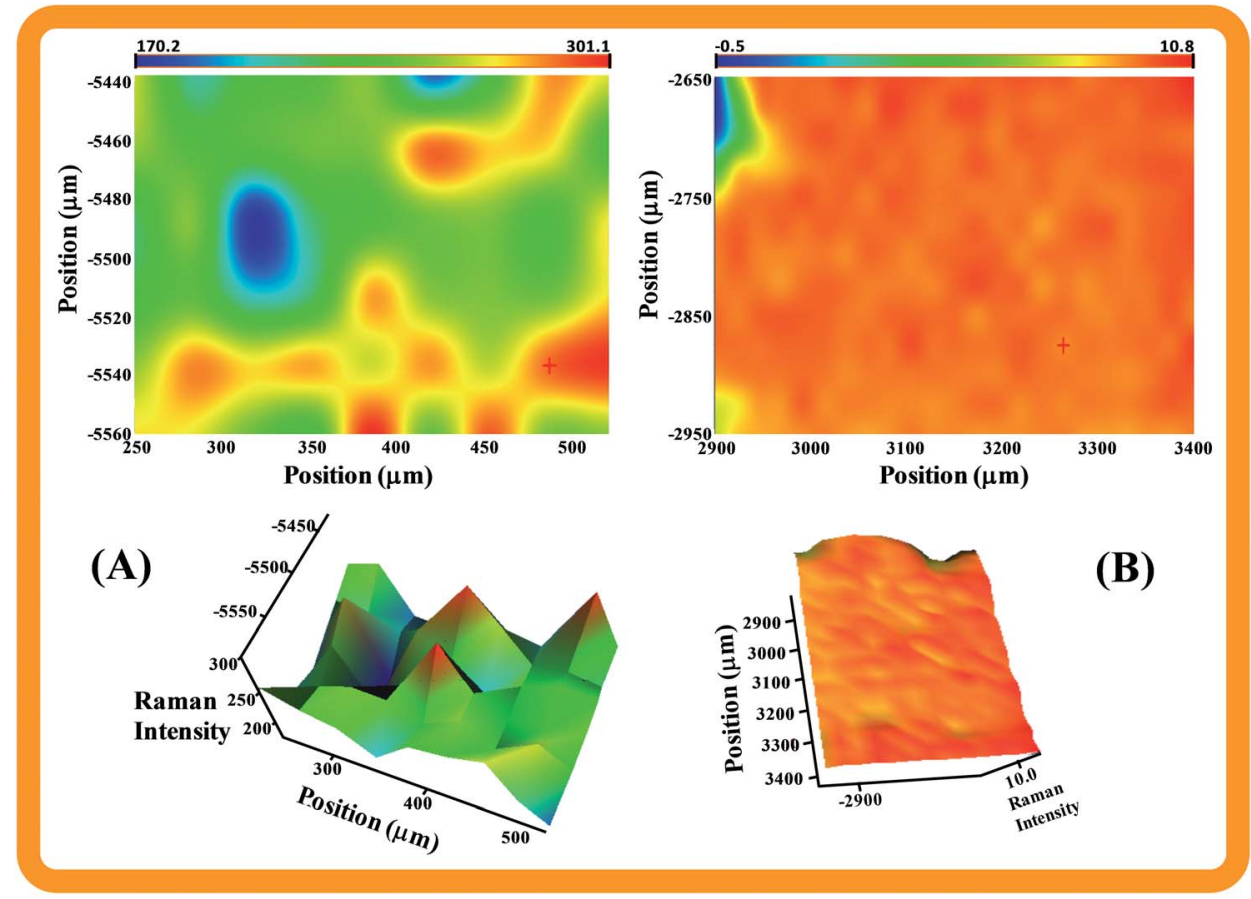

Fig. 5 Raman spectra mapping of (A) PANI/graphene-SPE and (B) anti-hCG/PANI/graphene-SPE, along with the corresponding surface spatial distribution (bottom 3D graphics).

generation accounting the residues in the protein structure. This implied the need of specific fluorescence correction, which is detrimental to the quality of the resulting spectra. Despite this, clear changes were observed at each stage of the modification process with each protein.

\subsection{Polyaniline and antibody coverage}

The antibody coverage over the surface was assessed by mapping a $610 \times 500 \mu \mathrm{m}$ area of the PANI/graphene-SPE and
anti-hCG/PANI/graphene-SPE devices using a Raman spectrometer coupled to confocal microscopy and an automated XY stage.

Fig. 5 shows a typical colour coverage distribution map after graphene PANI electropolymerization (left side). This is a spatial map of the intensity of the PANI-related Raman peak at 1663 $\mathrm{cm}^{-1}$ and indicates that $83 \%$ of the analysed area was covered with PANI, considering that each spectrum entering this calculation displayed a Raman shift at $1663 \mathrm{~cm}^{-1}$ of PANI spectra with Raman intensity higher than 90 (a.u.). This region

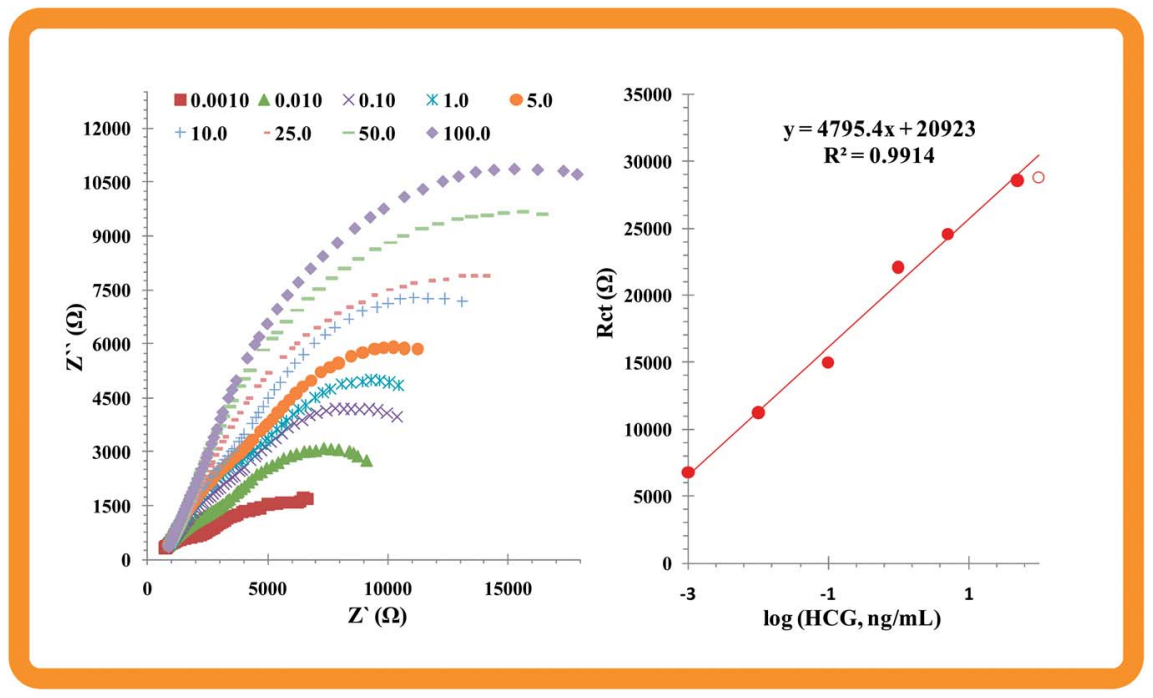

Fig. 6 Calibration curve of the immunosensor in real urine. Left: Nyquist plots of the BSA/anti-hCG/PANI/graphene-SPE sensor in a solution of $5.0 \mathrm{mM}\left[\mathrm{Fe}(\mathrm{CN})_{6}\right]^{3-}$ and $\left[\mathrm{Fe}(\mathrm{CN})_{6}\right]^{4-}$ in PBS buffer $\mathrm{pH}$ 6.5, after incubating the sensor in increasing concentrations of hCG (concentration levels indicated in the graphic, $\mathrm{ng} \mathrm{mL}^{-1}$ ). Right: $R_{\mathrm{ct}}$ values extracted from the Nyquist plots against log hCG concentration. 


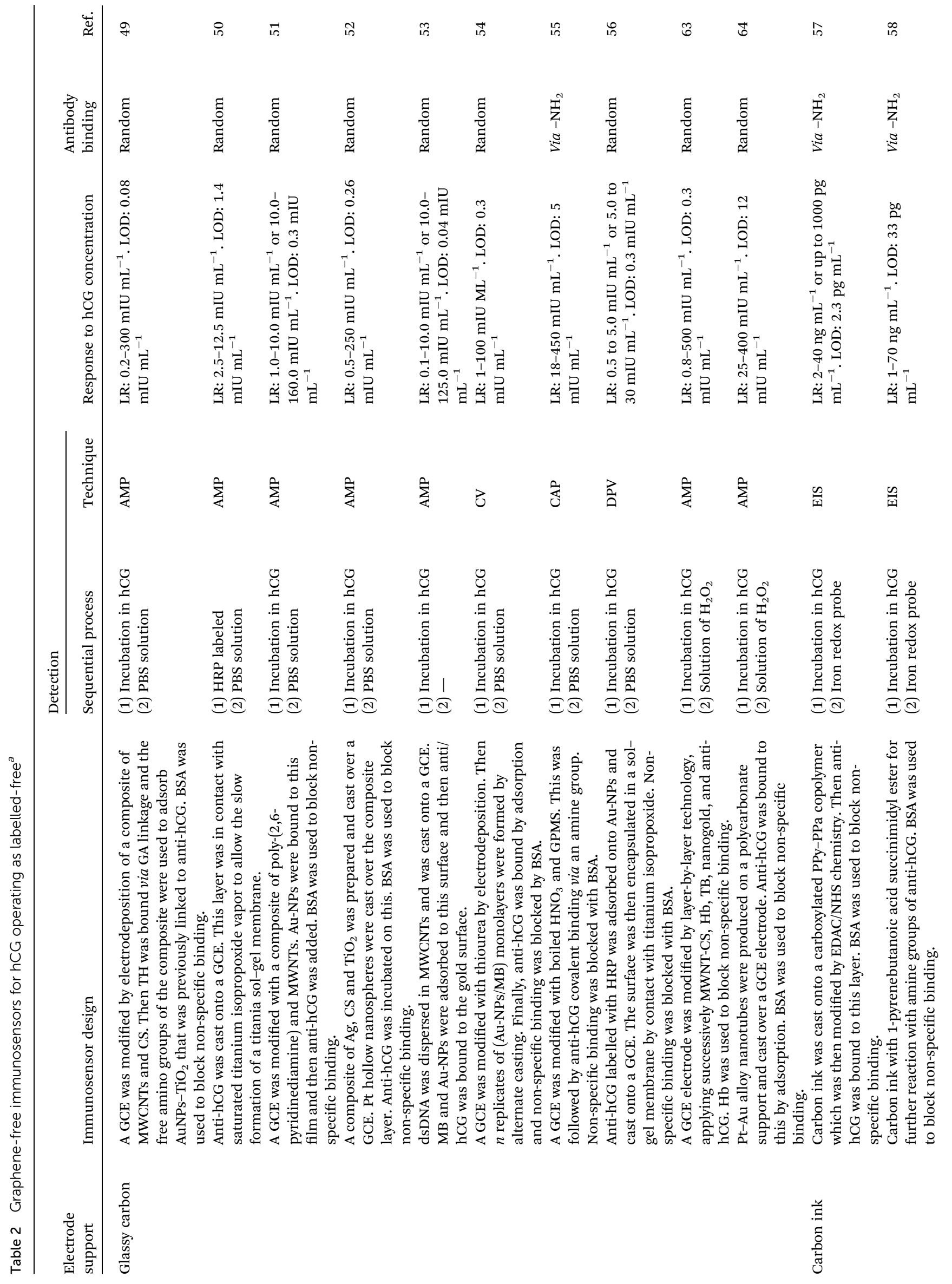




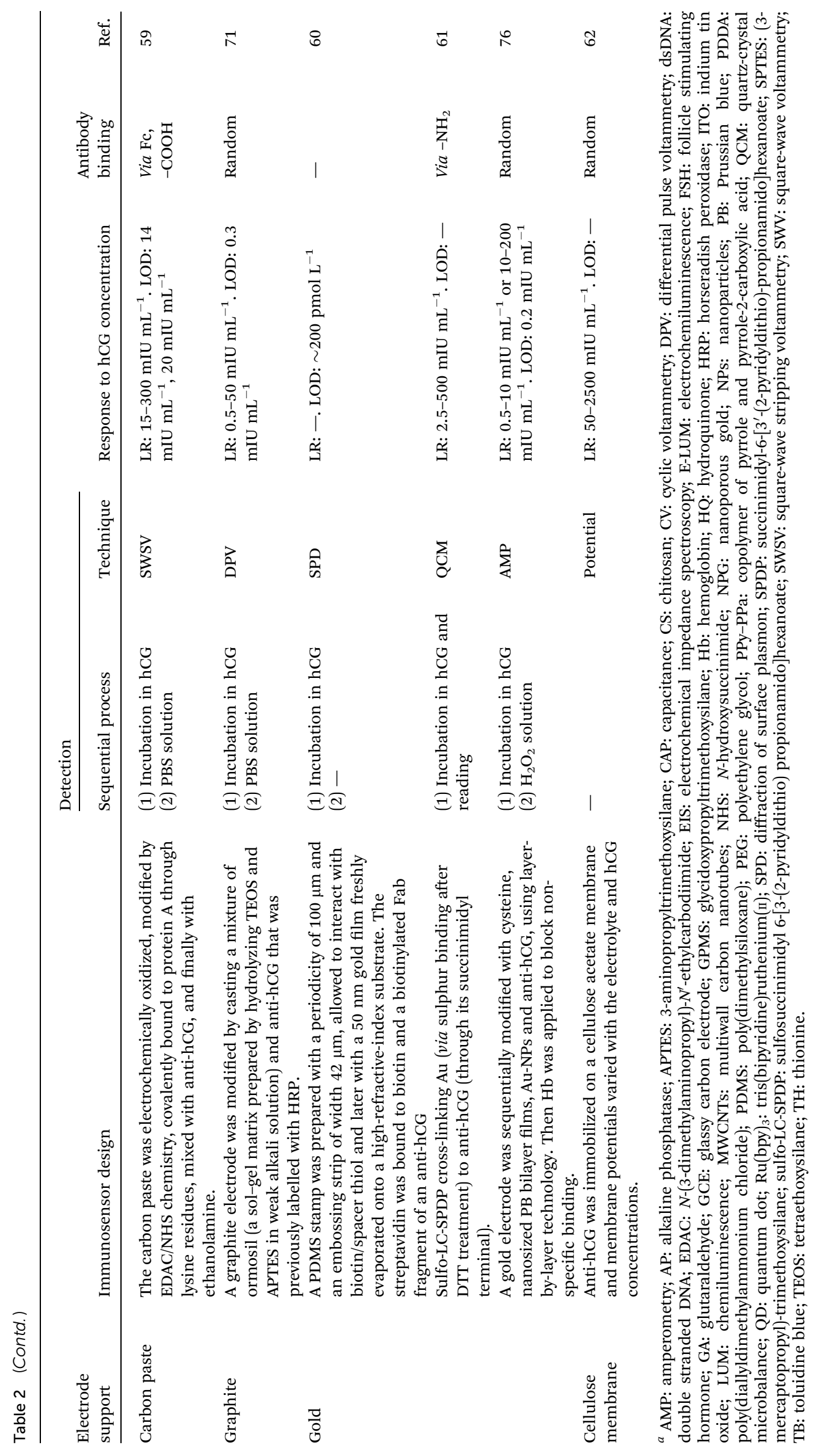




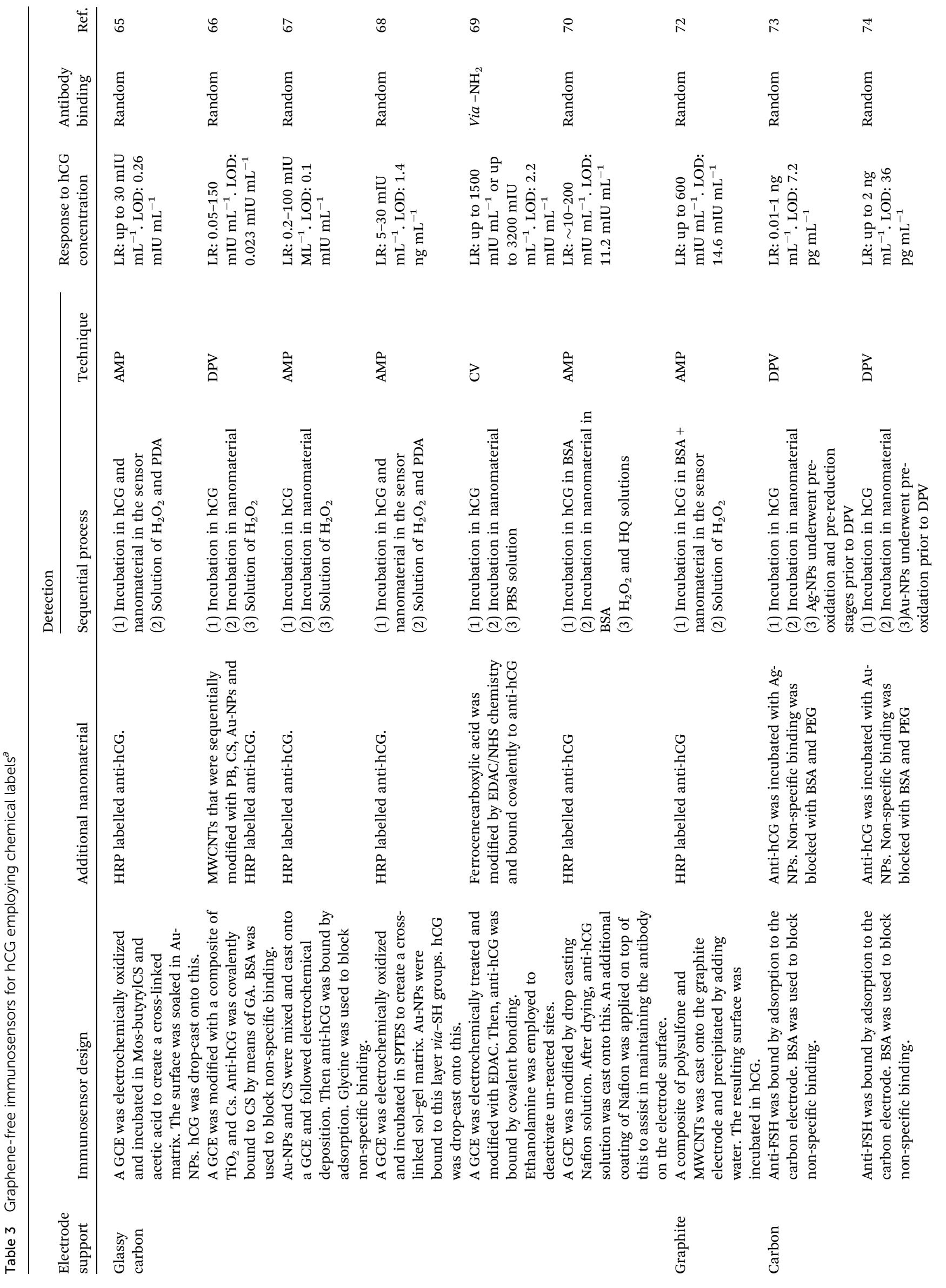




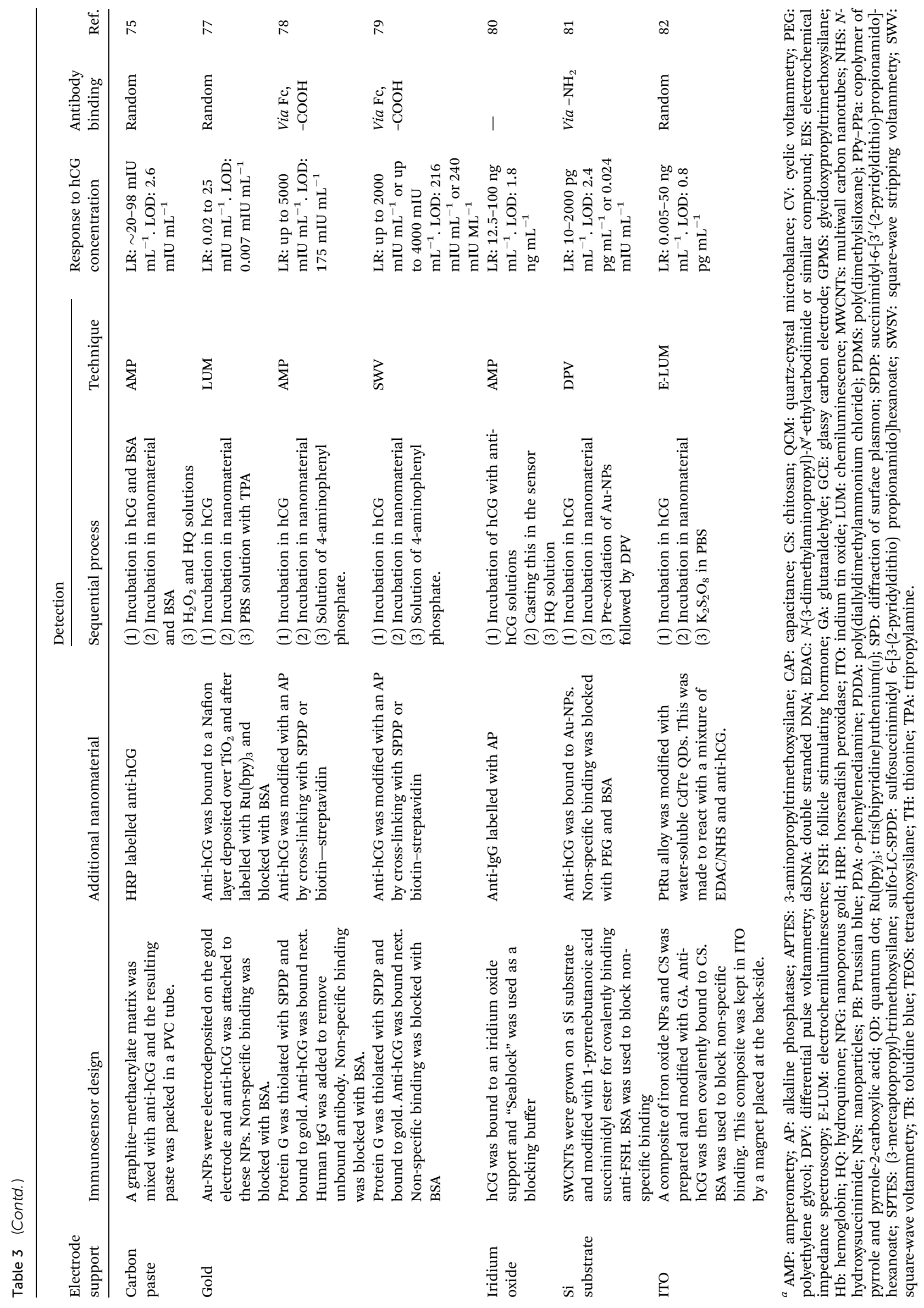


was used for being completely different from that of grapheneSPEs.

The colour coverage distribution map of anti-hCG/PANI/ graphene-SPE (right side) was obtained in a similar way, considering this time the intensities of Raman peaks at 1484 $\mathrm{cm}^{-1}$, because this was not present in graphene. The anti-hCG coverage of the surface was $97 \%$, extracted by spectral mapping of the Raman peaks at $1484 \mathrm{~cm}^{-1}$ with intensities greater than $27 \%$.

\subsection{Analytical performance}

Fig. 2C shows the Nyquist plots of the immunosensor Ab/PANI/ graphene-SPE against increasing hCG concentrations, while Fig. 2D shows the corresponding EIS calibration curve, plotting $R_{\text {ct }} \mathrm{Ab} / \mathrm{PANI} /$ graphene-SPE against the logarithm of hCG concentration. The concentration range of hCG used for calibration was 0.01 to $5 \mathrm{ng} \mathrm{mL}^{-1}$. No diffusion-controlled effect was observed in the EIS spectrum, and the diameter of the semicircle increased with increasing hCG concentrations. This suggests that a non-Faradaic process was present.

The $R_{\mathrm{ct}}$ in the Nyquist plot increased linearly with the increase of the logarithm of hCG concentration. This is as expected because protein structures bound to the surface of an electrode typically act as barriers to electrical transfer. The average slope of the $R_{\mathrm{ct}}$ versus $\log [\mathrm{hCG}]$ was $3.6 \mathrm{k} \Omega /\left[\mathrm{hCG}, \mathrm{ng} \mathrm{mL}^{-1}\right]$ with an $R^{2}$ correlation coefficient of 0.98 . The limit of detection (LOD) was determined to be $0.286 \mathrm{pg} \mathrm{mL}^{-1}$. This represents a significantly lower detection limit than those of previously reported graphene and anti-hCG sensors.

\subsection{Selectivity of the sensor}

Typically, selectivity is assessed by evaluating the effect of compounds present in the test solution, other than the target analyte (hCG), on the analytical response of a sensor device. To simulate the effect of real biological conditions, and ascertain the effect of urine on the performance of the sensor, the immunosensor was exposed to solutions of synthetic urine spiked with hCG. The selectivity of the sensor was thus investigated by monitoring its response to the synthetic urine. The synthetic urine was prepared according to recipes from ref. 48 and contained creatinine, magnesium chloride, calcium chloride, sodium dihydrogen phosphate, ammonium chloride, potassium sulphate and sodium chloride.

The EIS calibration curve of the immunosensor in response to increasing concentrations of hCG in synthetic urine was very similar to that of the sensor in PBS. Thus, no interference in the sensor, related to synthetic urine, was observed. This suggests that the sensor would be capable of selective and specific detection of hCG in real urine samples. The calibration curve was made for hCG concentrations ranging from 0.001 to $100 \mathrm{ng} \mathrm{mL}^{-1}$, showing

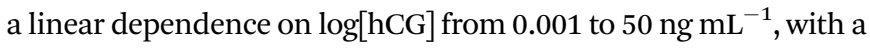
slope of $14.8 \mathrm{k} \Omega /\left[\mathrm{hCG}, \mathrm{ng} \mathrm{mL} \mathrm{m}^{-1}\right]$.

\subsection{Application to urine samples}

The values of hCG in real urine samples were determined by EIS measurements after previous calibration of the Ab/PANI/
graphene-SPE device. The typical Nyquist plots obtained are shown in Fig. 6, along with the corresponding calibration curve. The sensor exhibited linear behaviour from 0.001 to $50 \mathrm{ng}$ $\mathrm{mL}^{-1}$, when the standard hCG solutions were prepared in urine of non-pregnant women. When another sensor Ab/PANI/graphene-SPE was tested using urine of a pregnant woman, the obtained concentration was $63 \mathrm{ng} \mathrm{mL}^{-1}\left(315 \mathrm{mIU} \mathrm{mL}^{-1}\right)$, which corresponded to a pregnancy period lying within 3 weeks to 2 months, which is also in agreement with the patients term of pregnancy status. The format of the $R_{\mathrm{ct}}$ curve was close to that of the standards (Fig. 6), suggesting that the system was operating well when applied under real sample conditions.

\section{Conclusions}

In summary, a new impedimetric biosensor for hCG was fabricated based on antibody-antigen binding to a PANImodified screen-printed graphene electrode. In this work, it was shown that polyaniline was successfully deposited on graphene-SPE. The simple approach for antibody binding enabled attachment of the antibody to the sensing layer, and provided excellent reproducibility and stability. The electrode impedance increased linearly with increasing hCG protein concentrations and was not significantly affected by interference from chemical components present in synthetic urine.

Compared to other immunosensors that employ graphene (Table 1), this is the only device that uses graphene as a conductive support and preserves the native chemical structure of graphene that is responsible for its excellent electrical properties. This simple aspect may have contributed to the good analytical features achieved here, mostly regarding the wide linear range and the low lower limit of linear response.

Compared to other immunosensors presented in the literature, shown in Tables 2 and 3, ${ }^{49-82}$ the presented device offers a simple design, requiring only electropolymerization of aniline and subsequent binding of an antibody to this layer that has activated $-\mathrm{COOH}$ functions. This simple approach contributed as well to a suitable orientation of the antibodies over the platform, which may also have been a major contributor to the sensitivity of the immunosensor. An antibody should be bound to the sensing layer through its Fc terminal (that is composed of $-\mathrm{COOH}$ functions), ensuring that Fab regions (with $-\mathrm{NH}_{2}$ functions) remain free and accessible to antigens. Almost all previous studies give no importance to this feature, binding the antibody randomly, but some others use an amine function for this purpose, which means that the antibody is linked upsidedown. Only a few studies use specific proteins that show specific affinity to the Fc terminal antibody sites, ensuring that a suitable orientation is achieved, but this approach is however much more expensive than the one presented here and leads to a more complex immunosensor assembly.

The most complex processes presented in the literature are typically linked to the presence of chemical labels (Table 3 ). The presented labels involved usually an antibody species, which means that the final detection of hCG required an additional incubation period, besides that of the antigen. In the present work, the already simple device was coupled to EIS detection, 
avoiding the need for a chemical label, and thus leading to a simpler and quicker analytical procedure.

Overall, PANI modified graphene may also be a promising sensing platform for assembling other immunosensing devices. The sensor has been used to successfully detect hCG concentrations in the urine of pregnant women, which concurs with the pregnancy status. The presented results and the accuracy and precision of the analytical data from the antibody/PANI modified graphene sensors proposed in this work suggest that these sensors may be used in POC diagnostics in the near future.

\section{Acknowledgements}

This work is funded by the European Social Fund (ESF) through the European Union's Convergence programme administered by the Welsh Government, Knowledge Economy Skills Scholarships (KESS), EPSRC Project: EP/I00193X/1. The authors would also like to acknowledge IG innovations for supplying the anti-hCG antibodies and Thermo/Unicam for access to the Raman equipment.

\section{References}

1 J. Wu, Z. Fu, F. Yan and H. Ju, TrAC, Trends Anal. Chem., 2007, 26, 679-688.

2 D. R. Theavenot, K. Tóth, R. A. Durst and G. S. Wilson, Pure Appl. Chem., 1999, 71, 2333-2348.

3 S. I. Stoeva, J. S. Lee, J. E. Smith, S. T. Rosen and C. A. Mirkin, J. Am. Chem. Soc., 2006, 128, 8378-8379.

4 F. Darain, S. U. Park and Y. B. Shim, Biosens. Bioelectron., 2003, 18, 773-780.

5 Y. Takahashi, A. I. Shevchuk, P. Novak, Y. Murakami, H. Shiku, Y. E. Korchev and T. Matsue, J. Am. Chem. Soc., 2010, 132, 10118-10126.

6 H. J. Lee, S. H. Lee, T. Yasukawa, J. Ramón-Azcón, F. Mizutani, K. Ino, H. Shiku and T. Matsue, Talanta, 2010, 81, 657-663.

7 P. Martin, Mater. Today, 2011, 14, 308-315.

8 G. Liu and Y. Lin, Talanta, 2007, 74, 308-317.

9 S. Sánchez, M. Roldán, S. Pérez and E. Fàbregas, Anal. Chem., 2008, 80, 6508-6514.

10 N. Liu and X. Guo, NPG Asia Mater., 2012, 4, e23, DOI: 10.1038/am.2012.42.

11 A. Plume, es. Trends, 2010, 18, 5-7.

12 Y. Li, L. Tang and J. Li, Electrochem. Commun., 2009, 11, 846849.

13 J. Li, S. Guo, Y. Zhai and E. Wang, Electrochem. Commun., 2009, 11, 1085-1088.

14 D. Li, M. B. Muller, S. Gilje, R. B. Kaner and G. G. Wallace, Nat. Nanotechnol., 2008, 3, 101-105.

15 Q. Wei, R. Li, B. Du, D. Wu, Y. Han, Y. Cai, Y. Zhao, X. Xin, H. Li and M. Yang, Sens. Actuators, B, 2011, 153, 256-260.

16 R. McCreery, Chem. Rev., 2008, 108, 2646-2687.

17 M. Li, R. Li, C. Li and N. Wu, Frontiers in Bioscience, 2011, 3, 1308-1331.

18 Y. Shao, J. Wang, H. Wu, J. Liu, I. A. Aksay and Y. Lin, Electroanal., 2010, 22, 1027-1036.
19 T. Kuila, S. Bose, P. Khanra, A. Mishra, N. Kim and J. Lee, Biosens. Bioelectron., 2011, 26, 4637-4648.

20 F. Zhang, X. Chen, R. A. Boulos, F. Md Yasin, H. Lu, C. Raston and H. Zhang, Chem. Commun., 2013, 49, 4845-4847.

21 H. K. Hassan, N. F. Atta and A. Galal, Int. J. Electrochem. Sci., 2012, 7, 11161-11181.

22 Y. Wang, Z. Li, J. Wang, J. Li and Y. Lin, Trends Biotechnol., 2011, 29, 205-212.

23 K. Handschuh, J. Guibourdenche, V. Tsatsaris, M. Guesnon, I. Laurendeau, D. Evain-Brion and T. Fournier, Endocrinology, 2007, 148, 5011-5019.

24 U. H. Stenman, H. Alfthan and K. Hotakainen, Clin. Biochem., 2004, 37, 549-561.

25 H. Lund, S. B. Torsetnes, E. Paus, K. Nustad, L. Reubsaet and T. G. Halvorsen, J. Proteome Res., 2009, 8, 5241-5252.

26 T. K. Lim and T. Matsunaga, Biosens. Bioelectron., 2001, 16, 1063-1069.

27 J. Lu, S. Liu, S. Ge, M. Yan, J. Yu and X. Hu, Biosens. Bioelectron., 2012, 33, 29-35.

28 Q. Wei, R. Li, B. Du, D. Wu, Y. Han, Y. Cai, Y. Zhao, X. Xin, H. Li and M. Yang, Sens. Actuators, B, 2011, 153, 256-260.

29 D. Wu, Y. Zhang, L. Shi, Y. Cai, H. Ma, B. Du and Q. Wei, Electroanal., 2013, 25, 427-432.

30 R. Li, D. Wu, H. Li, C. Xu, H. Wang, Y. Zhao, Y. Cai, Q. Wei and B. Du, Anal. Biochem., 2011, 414, 196-201.

31 S. Bhadra, N. K. Singha and D. Khastgir, J. Chem. Eng. Mater. Sci., 2011, 2, 1-11.

32 Z. L. Wang, R. Guo, G. R. Li, H. L. Lu, Z. Q. Liu, F. M. Xiao, M. Zhang and Y. X. Tong, J. Mater. Chem., 2012, 22, 24012404.

33 D. Li, J. Huang and R. Kaner, Acc. Chem. Res., 2009, 42, 135145.

34 Z. Zheng, Y. Du, Q. Feng, Z. Wang and C. Wang, J. Mol. Catal. A: Chem., 2012, 353-354, 80-86.

35 H. D. Tran, D. Li and R. B. Kaner, Adv. Mater., 2009, 21, 14871499.

36 Y. Liu, R. Deng, Z. Wang and H. Liu, J. Mater. Chem., 2012, 22, 13619-13624.

37 K. Jiang, L. S. Schadler, R. W. Siegel, X. Zhang, H. Zhang and M. Terrone, J. Mater. Chem., 2004, 14, 37-39.

38 J. Daniels and N. Pourmand, Electroanal., 2007, 19, 12391257.

39 S. S. Srinivasan and P. C. Sharma, Development of Novel Polymer Nanostructures and Nanoscale Complex Hydrides for Reversible Hydrogen Storage, in Hydrogen Storage, ed. J. Liu, 2012, Ch 1, ISBN 978-953-51-0731-6, InTech.

40 N. Carrillo, U. León-Silva, T. Avalos, M. Nicho, S. Serna, F. Castillon, M. Farias and R. Cruz-Silva, J. Colloid Interface Sci., 2012, 369, 103-110.

41 J. Chen, J. Yang, X. Yan and Q. Xue, Synth. Met., 2010, 160, 2452-2458.

42 M. Trchová and A. J. Stejskal, Pure Appl. Chem., 2011, 83, 1803-1817.

43 D. C. Elias, R. R. Nair, T. M. G. Mohiuddin, S. V. Morozov, P. Blake, M. P. Halsall, A. C. Ferrari, D. W. Boukhvalov, M. I. Katsnelson, A. K. Geim and K. S. Novoselov, Science, 2010, 323, 610-613. 
44 J. Ding, M. Wang, X. Yan, X. Zhang, C. Ran, H. Chen and X. Yao, J. Colloid Interface Sci., 2013, 395, 40-44.

45 E. B. Barros, K. Sato, G. G. Samsonidze, A. G. Souza Filho, M. S. Dresselhaus and R. Saito, Phys. Rev. B: Condens. Matter Mater. Phys., 2011, 83(245435), 8, DOI: 10.1103/ PhysRevB.83.245435.

46 B. Shen, J. Ding, X. Yan, W. Feng, J. Li and Q. Xue, Appl. Surf. Sci., 2012, 258, 4523-4531.

47 A. Das, B. Chakraborty and A. K. Sood, Bull. Mater. Sci., 2008, 31, 579-584.

$48 \mathrm{H}$. Haddad, Synthetic urine for use in urine assay kits used for medical identification of illness or drug abuse, comprises a synthetic urine formulation and a heat activator, Patent Number: US2004077106-A1, 2004, US7109035-B2, 2006.

49 H. Yang, R. Yuan, Y. Chai and Z. Ying, Colloids Surf., B, 2011, 82(2), 463-469.

50 C. Jin, Y. Feng, D. Zong and J. Huangxian, Biosens. Bioelectron., 2005, 21, 330-336.

51 J. Wang, R. Yuan, Y. Chai, S. Cao, S. Guan, P. Fu and L. Min, Biochem. Eng. J., 2010, 51, 95-101.

52 H. Yang, R. Yuan, Y. Chai, Y. Zhuo and H. Su, J. Chem. Technol. Biotechnol., 2010, 85, 577-582.

53 S. Cao, J. Wang, R. Yuan and Y. Chai, Sens. Lett., 2011, 9, 1636-1642.

54 R. Chai, R. Yuan, Y. Chai, C. Ou, S. Cao and X. Li, Talanta, 2008, 74, 1330-1336.

55 J. Y. Liao, Appl. Microbiol. Biotechnol., 2007, 74, 13851391.

56 J. Chen, J. H. Tang, F. Yan and H. X. Ju, Biomaterials, 2006, 27, 2313-2321.

57 L. T. N. Truong, M. Chikaea, Y. Ukitaa and Y. Takamura, Talanta, 2011, 85(5), 2576-2580.

58 T. T. N. Lien, N. X. Viet, M. Chikae, Y. Ukita and Y. Takamura, J. Biosens. Bioelectron., 2011, 2(107), 6, DOI: 10.4172/2155-6210.1000107.

59 K. Kerman, N. Nagatani, M. Chikae, T. Yuhi, Y. Takamura and E. Tamiya, Anal. Chem., 2006, 78, 5612-5616.

60 F. Yu and W. Knoll, Anal. Chem., 2004, 76, 1971-1975.

61 B. Zhanga, Q. Mao, X. Zhang, T. Jiang, M. Chen, F. Yu and W. Fu, Biosens. Bioelectron., 2004, 19, 711-720.
62 M. Lu, D. Fang, J. Zhang and K. Chen, Acta Phys. Sin., 1992, 8, 523-526.

63 N. Li, R. Yuan, Y. Chai and S. Chen, Bioprocess Biosyst. Eng., 2008, 31, 551-558.

64 M. Tao, X. Li, Z. Wu, M. Wang, M. Hua and Y. Yang, Clin. Chim. Acta, 2011, 412, 550-555.

65 C. Jin, Y. Feng, D. Dan, W. Jie and J. Huangxian, Electroanal., 2006, 18(7), 670-676.

66 H. Yang, R. Yuan, Y. Chai, H. Su, Y. Zhuo, W. Jiang and Z. Song, Electrochim. Acta, 2011, 56, 1973-1980.

67 G. Yang, Y. Chang, H. Yang, L. Tan, Z. Wu, X. Lu and Y. Yang, Anal. Chim. Acta, 2009, 644, 72-77.

68 J. Chen, F. Yan, F. Tan and H. Ju, Electroanal., 2006, 18, 1696-1702.

69 M. Akram, M. C. Stuart and D. K. Y. Wong, Electroanal., 2006, 18, 237-246.

70 A. F. Chetcuti, D. K. Y. Wong and M. C. Stuart, Anal. Chem., 1999, 71, 4088-4094.

71 F. Tan, F. Yan and H. Ju, Biosens. Bioelectron., 2007, 22, 29452951.

72 S. Sanchez, M. Roldan, S. Perez and E. Fabregas, Anal. Chem., 2008, 80, 6508-6514.

73 M. Chikae, K. Idegami, N. Nagatani, E. Tamiya and Y. Takamura, Electrochem., 2010, 78, 748-753.

74 K. Idegami, M. Chikae, K. Kerman, N. Nagatani, T. Yuhi, E. Endo and E. Tamiya, Electroanal., 2008, 20, 14-21.

75 M. Santandreu, S. Alegret and E. Fabregas, Anal. Chim. Acta, 1999, 396, 181-188.

76 J. Wang, R. Yuan, Y. Chai, B. Yin, Y. Xu and S. Guan, Electroanal., 2009, 21, 707-714.

77 L. Mao, R. Yuan, Y. Chai, Y. Zhuo and X. Yang, Sens. Actuators, B, 2010, 149, 226-232.

78 J. M. Fowler, M. C. Stuart and D. K. Y. Wong, Electrochem. Commun., 2008, 10, 1020-1023.

79 J. A. Fowler, M. C. Stuart and D. K. Y. Wong, Biosens. Bioelectron., 2007, 23, 633-639.

80 M. S. Wilson and W. Nie, Anal. Chem., 2006, 78, 6476-6483.

81 Y. Zhang, S. Ge, S. Wang, M. Yan, J. Yu, X. Song and W. Liu, Biosens. Bioelectron., 2013, 42, 592-597.

82 Y. Zhang, S. Ge, S. Wang, M. Yan, J. Yu, X. Song and W. Liu, Analyst, 2012, 137, 2176-2182. 